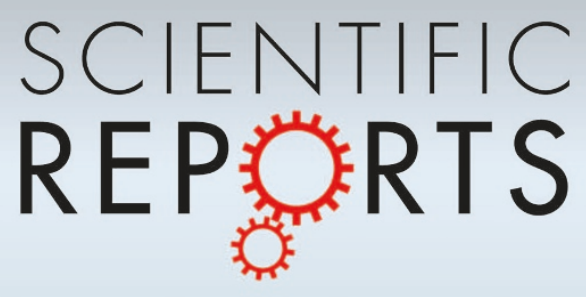

OPEN

CONFERENCE

PROCEEDINGS

ISFM2014

SUBJECT AREAS:

NANOPARTICLES

MAGNETIC PROPERTIES AND MATERIALS

Received

12 October 2014

Accepted

23 January 2015

Published

23 March 2015

Correspondence and requests for materials should be addressed to L.Z. (stszhl@mail.sysu. edu.cn)

\title{
Preparation and Characterization of
} $\mathrm{Fe}_{3} \mathrm{O}_{4}$ Particles with Novel Nanosheets Morphology and Magnetochromatic Property by a Modified Solvothermal
Method

\author{
Lin Zhuang' ${ }^{1}$ Wei Zhang ${ }^{2}$, Yongxin Zhao ${ }^{1,3}$, Hui Shen $^{3}$, Han Lin $^{4}$ \& Jinhua Liang ${ }^{1,3}$
}

\begin{abstract}
'State Key Laboratory of Optoelectronic Materials and Technologies, Guangdong Provincial Key Laboratory of Photovoltaics Technologies, School of Physics and Engineering, Sun Yat-Sen University, Guangzhou 510275, P. R. China, ${ }^{2}$ School of Physics and Optoelectronic Engineering, Guangdong University of Technology, Guangzhou 510006, China, ${ }^{3}$ School of Chemistry and Chemical Engineering, Sun Yat-Sen University, Guangzhou 510275, P. R. China, ${ }^{4}$ Department of Orthodontics, Guanghua School of Stomatology, Hospital of Stomatology, Sun Yat-sen University, Guangzhou 510055, P.R. China.
\end{abstract}

Novel-morphological $\mathrm{Fe}_{3} \mathrm{O}_{4}$ nanosheets with magnetochromatic property have been prepared by a modified solvothermal method. Such nanosheets could form one-dimension photonic crystal under an external magnetic field. The $\mathrm{Fe}_{3} \mathrm{O}_{4}$ nanosheets suspension could strongly diffract visible light and display varied colors with changing the intensity of the magnetic field. The photonic response is rapid, fully reversible and widely tunable in the entire visible spectrum. Excellent magnetic properties of these $\mathrm{Fe}_{3} \mathrm{O}_{4}$ nanosheets are exhibited with a high saturation magnetization $(82.1 \mathrm{emu} / \mathrm{g})$, low remanence $(13.85 \mathrm{emu} / \mathrm{g})$ and low coercive force $(75.95 \mathrm{Oe})$. The amount of the solvent diethylene glycol (DEG) plays a key role in the formation of the sheet-shaped morphology. When the ratio of the DEG reaches $100 \%$, the growing of the crystal plane (111) of $\mathrm{Fe}_{3} \mathrm{O}_{4}$ is inhibited and the sheet-like $\mathrm{Fe}_{3} \mathrm{O}_{4}$ crystals are formed.

$\mathrm{N}$ anostructure magnetic $\mathrm{Fe}_{3} \mathrm{O}_{4}$ particles are of much interest because of their applications in a variety of areas such as ferrofluid, advanced magnetic materials, magnetic recording media and biomedical purpose, etc ${ }^{1-7}$. With the advancement of magnetic materials, especially in the area of the magnetic responsive photonic crystal, considerable efforts have been attempted to improve their magnetic and optical properties. Different from other external stimuli systems of responsive photonic crystal, introducing magnetic components into the photonic crystal makes it more convenient and precise to control the properties through an external magnetic field. Such magnetic responsive photonic crystals have been demonstrated in the applications of different color display units including dynamic displays, rewritable photonic papers and full color printing systems.

Recently, a high-temperature hydrolysis method was reported to synthesize monodisperse superparamagnetic $\mathrm{Fe}_{3} \mathrm{O}_{4}$ particles with the nanosphere morphology ${ }^{7}$. These $\mathrm{Fe}_{3} \mathrm{O}_{4}$ nanospheres could form one-dimension photonic crystal in aqueous and non-polar media, which could diffract the light of visible wavelengths. As Known,the magnetic properties of nanoparticles are greatly dependent on their morphologies. Varied morphologies of $\mathrm{Fe}_{3} \mathrm{O}_{4}$ nanocrystals including nanotubes ${ }^{8}$, hollow spheres ${ }^{9}$, nanochains ${ }^{10}$, nanoflowers ${ }^{11}$ and nanosheets ${ }^{12}$ have been fabricated. Compared to magnetite particles with other shapes, nanosheets have better crystallinity and smaller lattice distortion, which enable them possess better physical and magnetic properties. To the best of our knowledge, the synthesis of $\mathrm{Fe}_{3} \mathrm{O}_{4}$ nanosheets with the magnetochromatic property has hardly been reported.

Numerous chemical methods including microemulsions ${ }^{13}$, sol-gel syntheses ${ }^{14}$, sonochemical reactions ${ }^{15}$, hydrothermal reactions ${ }^{16}$, hydrolysis and thermolysis of precursors ${ }^{17}$ have been used to synthesize $\mathrm{Fe}_{3} \mathrm{O}_{4}$ magnetic nanoparticles. In the specific synthesis of nanosheets, the methods of solvothermal process ${ }^{12}$, solid-state thermal decomposition route ${ }^{18}$, supercritical fluid technique ${ }^{19}$ and bottom-up technique ${ }^{20}$ have been attempted. These synthesis processes are usually involved in one-step reaction, and the surface modification procedure is 


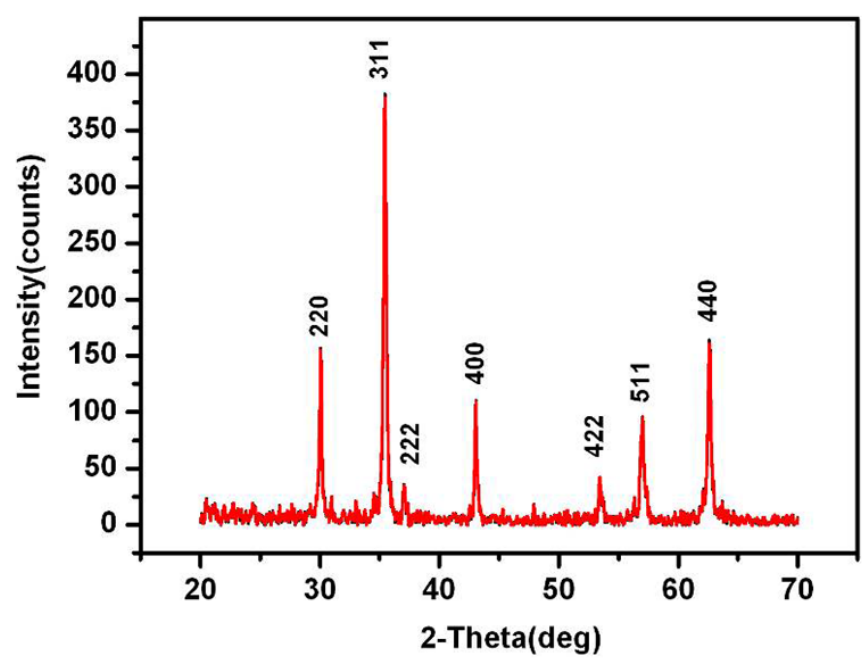

Figure $1 \mid \mathrm{XRD}$ pattern of the $\mathrm{Fe}_{3} \mathrm{O}_{4}$ nanosheets.

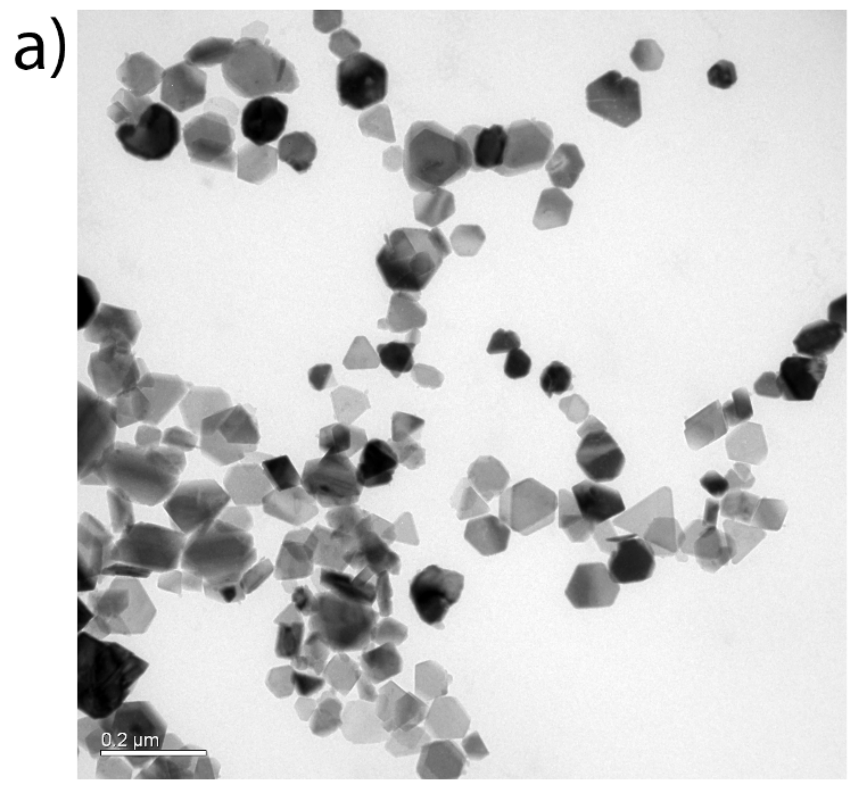

incorporated with the synthesis of particles. In addition, in the conventional synthesis methods, deoxygenated protection and precisely control of the $\mathrm{pH}$ value are required.

Here we report a two-step solvothermal method to synthesize the $\mathrm{Fe}_{3} \mathrm{O}_{4}$ nanosheets with good magnetochromatic property without deoxygenated protection and controlling of the $\mathrm{pH}$ value. In the process, the surface modification of the $\mathrm{Fe}_{3} \mathrm{O}_{4}$ particles is performed after synthesizing the particles. The separation of these two steps simplifies our synthesis method and increases the reproducibility of the obtained nanosheets. In addition, there are more choices for capping agents since the modification step is independent. When an external magnetic field is introduced to the $\mathrm{Fe}_{3} \mathrm{O}_{4}$ nanosheets suspension, the particles suspension could diffract light with different wavelength and display various colors under magnetic fields with different intensity. The photonic response of the magnetic $\mathrm{Fe}_{3} \mathrm{O}_{4}$ nanosheets is reversible and widely tunable in the visible spectrum, indicating great potential applications in sensors and optical devices.

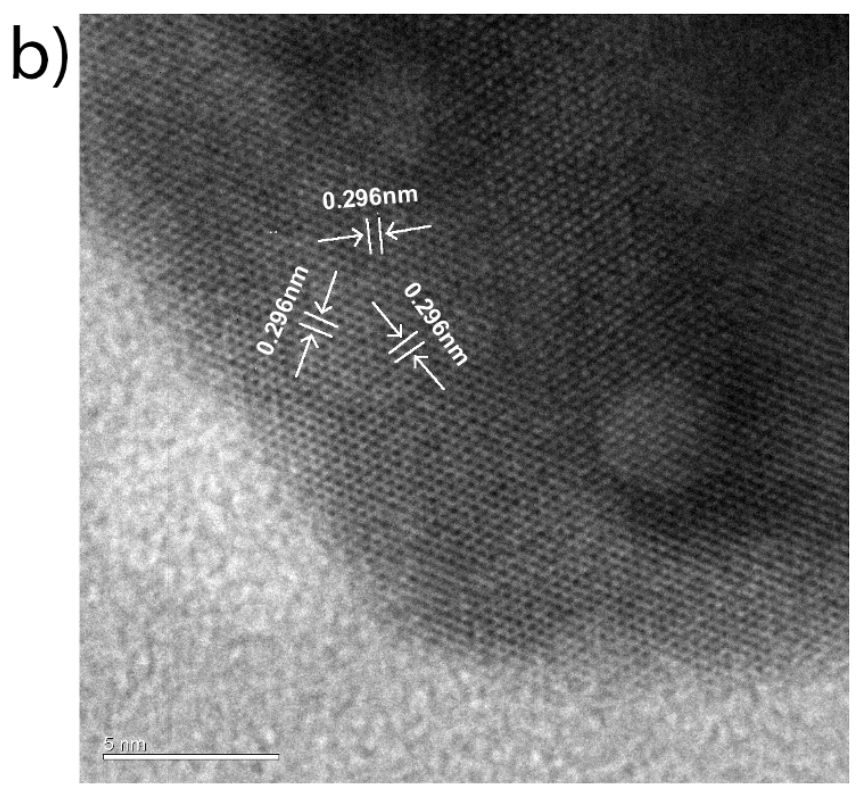

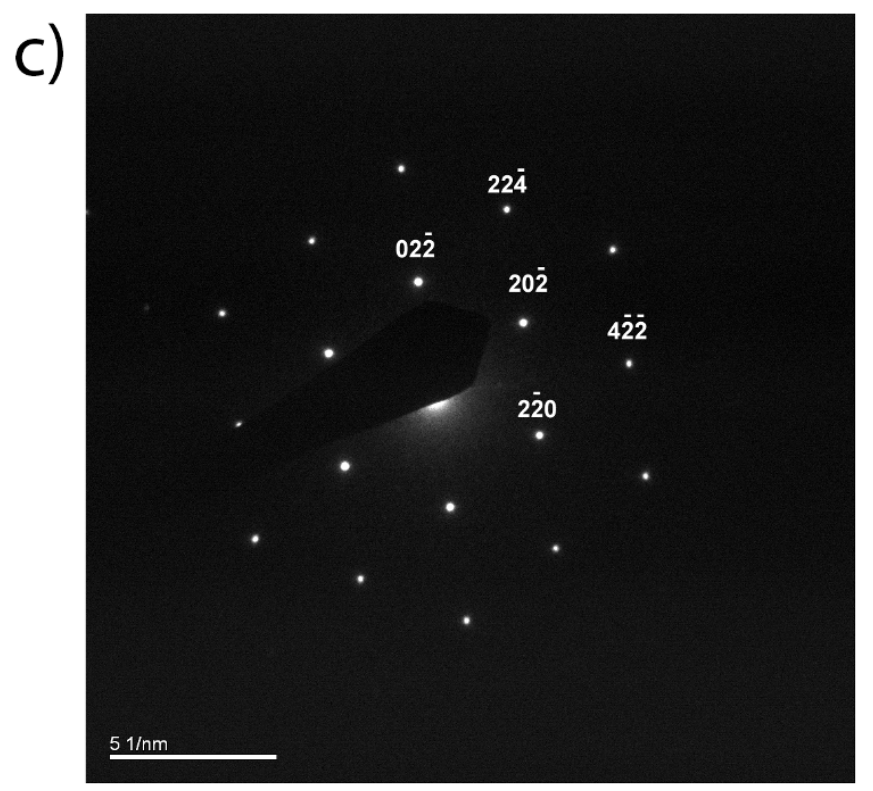

Figure $2 \mid$ (a) Low magnitude TEM image of $\mathrm{Fe}_{3} \mathrm{O}_{4}$ nanosheets; (b) HRTEM of $\mathrm{Fe}_{3} \mathrm{O}_{4}$ nanosheets; (c) SAED pattern of $\mathrm{Fe}_{3} \mathrm{O}_{4}$ nanosheets. The scale bars are $0.2 \mu \mathrm{m}, 5 \mathrm{~nm}$, and $51 / \mathrm{nm}$ respectively. 


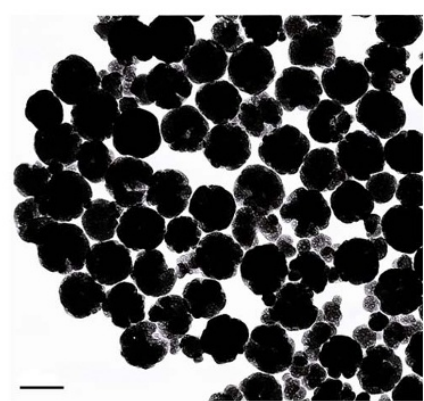

a

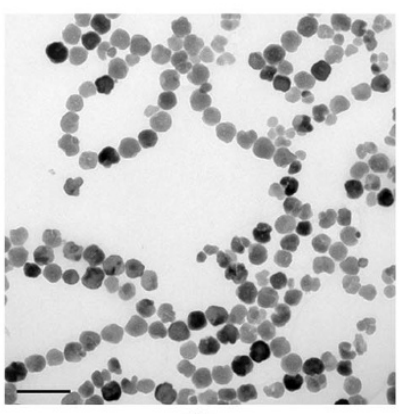

C

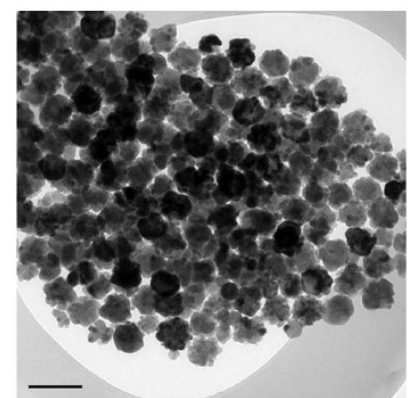

b

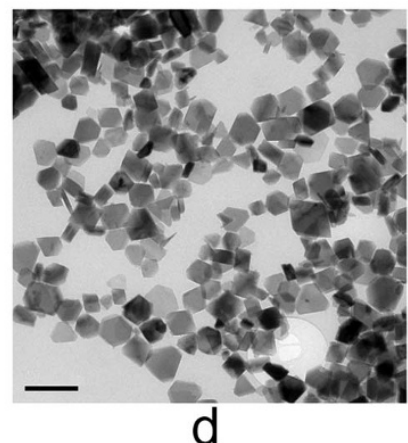

Figure 3 Low magnitude TEM images of $\mathrm{Fe}_{3} \mathrm{O}_{4}$ particles: (a) $180 \mathrm{~nm}$ particles $(\mathrm{DEG} / \mathrm{EG}=0 / 40)$; (b) $100 \mathrm{~nm}$ particles $(\mathrm{DEG} / \mathrm{EG}=26 / 14)$;

(c) $60 \mathrm{~nm}$ particles $(\mathrm{DEG} / \mathrm{EG}=30 / 10) ;(\mathrm{d})$ nanosheets $(\mathrm{DEG} / \mathrm{EG}=40 /$ 0). All the scale bars are $200 \mathrm{~nm}$.

\section{Results and Discussion}

The X-ray diffraction pattern of the $\mathrm{Fe}_{3} \mathrm{O}_{4}$ nanosheets is shown in Fig. 1, which reveals the formation of magnetite with well-defined crystallinity via the modified solvothermal process. All of the diffraction peaks match well with the normal characteristic diffractions of the $\mathrm{Fe}_{3} \mathrm{O}_{4}$ inverse spinel structure (PCPDFWIN v.2.02, PDF No. 890691). The calculated cell parameter $(8.3915 \AA)$ is comparable with the reported value. No obvious XRD peaks arising from impurities are found, which indicates no evidence that $\mathrm{Fe}_{3} \mathrm{O}_{4}$ particles are contaminated by foreign materials in the system.

In Fig. 2(a), the $\mathrm{Fe}_{3} \mathrm{O}_{4}$ nanosheets are of regular hexagonal or triangle structure with the edge length of $80-150 \mathrm{~nm}$. The detailed crystalline information of the particles is shown in Fig. 2(b) and Fig. 2(c). The HRTEM image of selected area in the sample in Fig. 2(b) indicates that the nanosheets have well-defined crystallinity

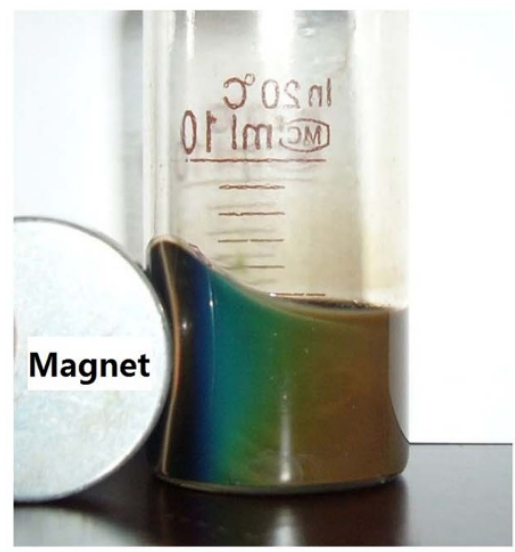

a

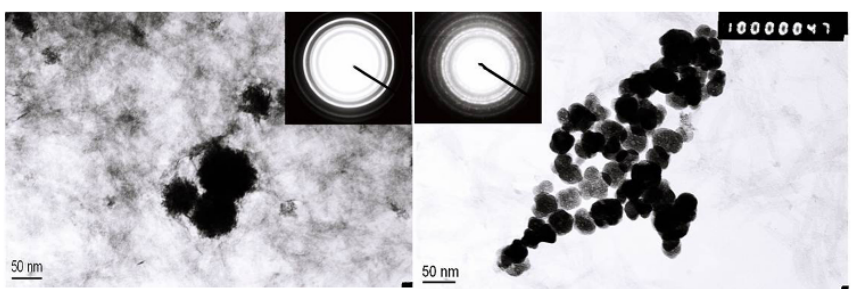

a

b
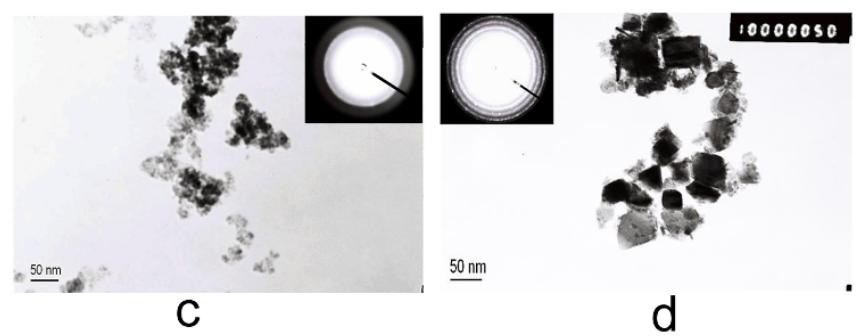

Figure $4 \mid$ Low magnitude TEM images of $\mathrm{Fe}_{3} \mathrm{O}_{4}$ product under the conditions of different reaction time and percentage of DEG in the solvent: (a) 1 hour, DEG\% = 65\%; (b) 3 hours, DEG\% = 65\%; (c) 1 hour, DEG $\%=100 \%$; (d) 3 hours, DEG\% $=100 \%$.

with an interplanar spacing of $0.296 \mathrm{~nm}$. The diffraction points array tidily along a certain direction in the selected area electron diffraction (SAED) pattern as shown in Fig. 2(c). Crystal planes of (220) and (422) could be found in Fig. 2(c), which are consistent with the XRD pattern in Fig. 1. From Fig. 2(b) and Fig. 2(c), it's clear that the growth rate of the crystal plane (111) is depressed, which is the key factor for the formation of the hexagonal or triangle shape of the nanosheets.

The nucleation of the $\mathrm{Fe}_{3} \mathrm{O}_{4}$ particles could be well controlled by changing the volume ratios of the solvent DEG and Ethylene Glycol (EG). The amount of the DEG plays a key role in the formation of the sheet-shaped $\mathrm{Fe}_{3} \mathrm{O}_{4}$ nanostructures, which can be confirmed by the comparison experiments with different concentrations of DEG. Fig. 3 shows that when the volume ratios of DEG/EG (v/v in $\mathrm{mL})$ are $0 / 40,26 / 14,30 / 10$ and $40 / 0$, different sizes and shapes of $\mathrm{Fe}_{3} \mathrm{O}_{4}$ particles have been obtained. The diameters of the nanospheres are $180 \mathrm{~nm}$ (Fig. 3a), $100 \mathrm{~nm}$ (Fig. 3b) and $60 \mathrm{~nm}$ (Fig. 3c), and the shape of the nanosheet is shown in Fig. $3 \mathrm{~d}$. The particle size decreases as the ratio of DEG/EG increases. This size tunability may due to the different diffusion coefficient of the ions in the solution caused by the varying concentrations of the DEG. Since DEG has a stronger affinity to the $\mathrm{Fe}^{3+}$ ions, the viscosity of the solvent increases as the portion of

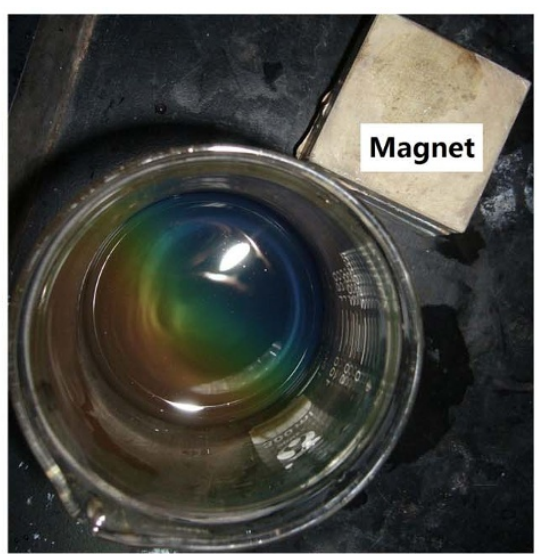

b

Figure $5 \mid$ Photographs of $\mathrm{Fe}_{3} \mathrm{O}_{4}$ nanosheets colloidal solution formed in response to an external magnetic field. (a) and (b), the external magnetic field are both set beside the $\mathrm{Fe}_{3} \mathrm{O}_{4}$ nanosheets suspension. 
the DEG increases and therefore the diffusion coefficient of the ions in the reaction system decreases, which leads to smaller grain size of the $\mathrm{Fe}_{3} \mathrm{O}_{4}$ particles and inhibits the formation of the secondary structure. In the solvothermal process, when the ratio of the DEG is rather low, the formation of the microstructure features after fast nucleation in solution relates to the aggregation growth process and the Ostwald ripening process. When the ratio of the DEG reaches $100 \%$, the mechanism of the crystal growth is different from the former and the morphology of the final product becomes nanosheet.

To further understand the formation mechanism of the $\mathrm{Fe}_{3} \mathrm{O}_{4}$ nanosheets, a number of matrix precursor experiments were carried out under different reaction time and different concentrations of DEG. TEM images in Fig. 4(a) and Fig. 4(c) reveal that the samples consist of unconsolidated particles when the reaction time is $1 \mathrm{~h}$. These unconsolidated particles only congregate but not form the crystal structure according to the electron diffraction patterns in Fig. 4(a) and Fig. 4(c). Fig. 4(b) reveals that the nanospheres preliminarily form after reaction for $3 \mathrm{~h}$. In the reaction system, the Fe (III) compounds hydrolyze and deoxidize to $\mathrm{Fe}(\mathrm{II})$ compounds partially. $\mathrm{Fe}_{3} \mathrm{O}_{4}$ crystal nucleus are formed by the interaction of $\mathrm{Fe}(\mathrm{II})$ and $\mathrm{Fe}$ (III) hydrates. The growing of the crystal follows the process of homogeneous nucleation and the final product of $\mathrm{Fe}_{3} \mathrm{O}_{4}$ nanospheres is obtained. Fig. 4(d) shows the formation of the nanosheets. When the ratio of the DEG reaches $100 \%$, DEG and $\mathrm{Fe}^{3+}$ ions complex and form a steady solution. The growing of the crystal and aggregation of nanoparticles are slow under the strong complexation and the high viscosity of the solvent. The growth of the crystal plane (111) is limited and the shape of the nanosheets, but not the nanospheres, is formed.

The $\mathrm{Fe}_{3} \mathrm{O}_{4}$ sheet-like nanoparticles exhibit good magnetochromatic properties. The photographs and reflection spectra of the aqueous solution of $\mathrm{Fe}_{3} \mathrm{O}_{4}$ colloidal particles (ca. $15.00 \mathrm{mg} \mathrm{mL} \mathrm{m}^{-1}$ ) in response to an external magnetic field are shown in Fig. 5 and Fig. 6. When a magnetic field is applied to the nanosheets suspension, the magnetic colloid displays clearly a gradient of colors. The particles in the suspension form a chain-like structure along with the direction of the external magnetic field, which is known as photonic $\mathrm{crystal}^{21}$. The structure formed by the magnetic nanosheets could diffract visible light of different wavelength ranging from 480 to $640 \mathrm{~nm}$ as shown in Fig. 6. The reflection rate increases and the diffraction peaks blue shift as the magnetic field is increased from 20 to $327 \mathrm{G}$. The low reflection rate and broad peak width at a low

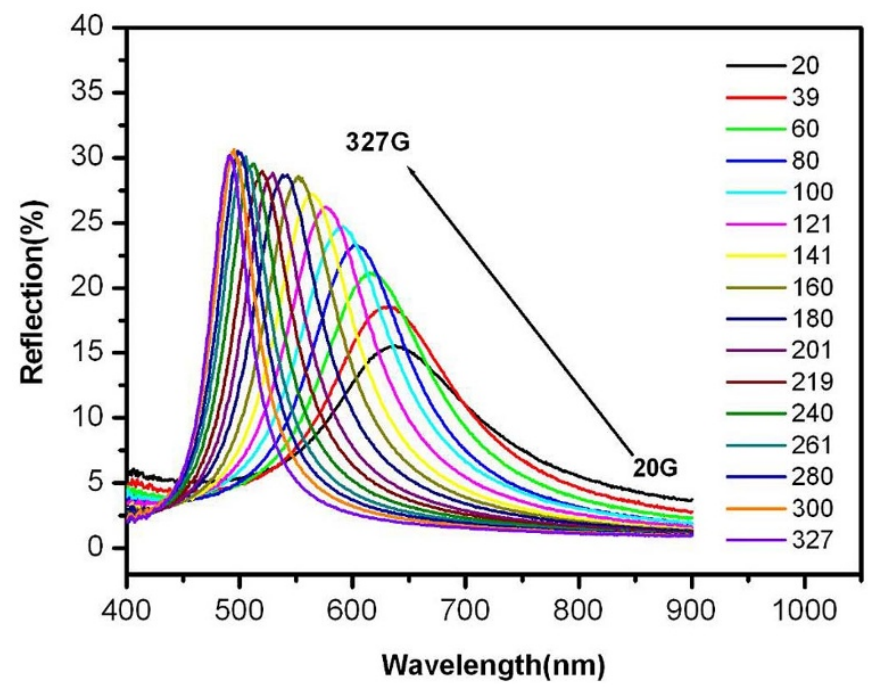

Figure 6 Reflection spectra of one-dimension photonic crystals formed by $\mathrm{Fe}_{3} \mathrm{O}_{4}$ nanosheets in aqueous solution in response to external magnetic field with different intensity. magnetic field are due to the Brownian motion, which significantly interferes with the formation of the long-range order structure. When the magnetic field increases, the magnetic interaction between two magnetic nanoparticles surpasses the Brownian motion and the chain-like structure is enhanced, which makes the diffraction peak sharper and higher.

The magnetic properties of the modified nanosheets and the corresponding nanosheets suspension were investigated respectively. As shown in Fig. 7, the $\mathrm{Fe}_{3} \mathrm{O}_{4}$ nanosheets exhibit soft magnetism with a low remanence $(13.85 \mathrm{emu} / \mathrm{g})$ and low coercive force $(75.95 \mathrm{Oe})$. The saturation magnetization of the magnetic nanosheets is $82.10 \mathrm{emu} / \mathrm{g}$, which is much higher than that of the nanoparticles prepared by the common chemical coprecipitation method (about 60-65 emu/g). The high reaction temperature in our modified solvothermal method results in a higher crystallinity and consequently leads to a higher saturation magnetization. While in the observation of the hysteresis loop of the particles suspension as shown in Fig. 8, the colloid exhibits essentially no remanence or coercive force. This interesting result explodes the previous conclusion that ferromagnetic particles cannot be dispersed in aqueous media. The remanence and the coercive force of the $\mathrm{Fe}_{3} \mathrm{O}_{4}$ nanosheets dispersed in aqueous solution are found to decrease to $0.042 \mathrm{emu} / \mathrm{g}$ and $1.94 \mathrm{Oe}$, respectively. This phenomenon indicates that if suitable surface modification is performed to decrease the magnetic force between the adjacent nanosheets, the ferromagnetic particles can rely on the Brownian motion to disrupt the chain-like structure induced by the magnetic moment, and then a transformation from ferromagnetism of the particles to a hysteresis loop with much small remanence and coercive force of the particles suspension appears. In this case, there will be no significant magnetic interactions remaining after removing the external magnetic field, which is important for achieving a reproducible and reversible optical response. The hysteresis loop of Fig. 8 displays irreversibility when the applied external magnetic field is between 100 and $900 \mathrm{G}$. when the external field is decreased from $900 \mathrm{G}$ in the upper curve, the particles could still keep the saturation magnetization until the field is decreased to be about $700 \mathrm{G}$. And during the magnetic field is between 900 and $100 \mathrm{G}$, the magnetization value in the upper curve is clearly larger than that in the bottom curve. This is due to the complex effect of metastable state of the magnetic dynamic nanoparticles and the possible relaxation characteristic of the formed chain-like structure when decreasing the external magnetic field, the Brownian dynamics of particles, and the electrostatic interactions due to the PAA.

In summary, magnetic $\mathrm{Fe}_{3} \mathrm{O}_{4}$ nanosheets have been synthesized by a two-step solvothermal method. The $\mathrm{Fe}_{3} \mathrm{O}_{4}$ nanosheets suspension exhibits magnetochromatic property under an external magnetic

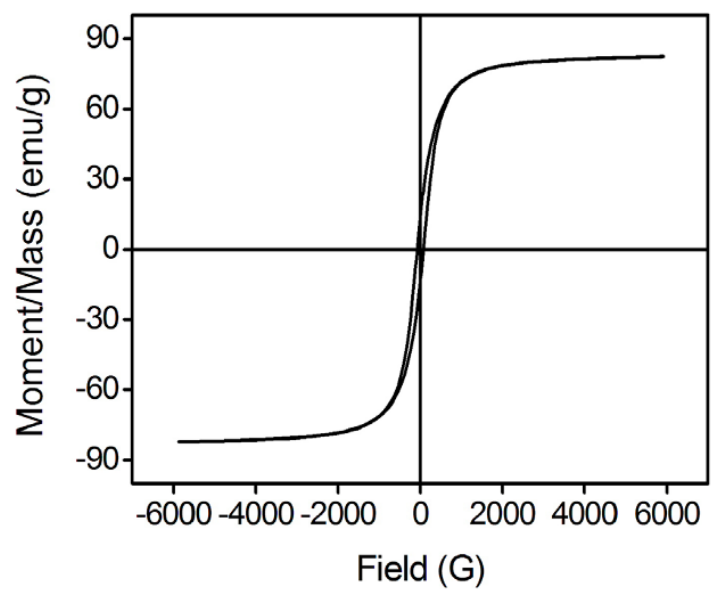

Figure 7 Magnetization curve of $\mathrm{Fe}_{3} \mathrm{O}_{4}$ nanosheets at room temperature. 


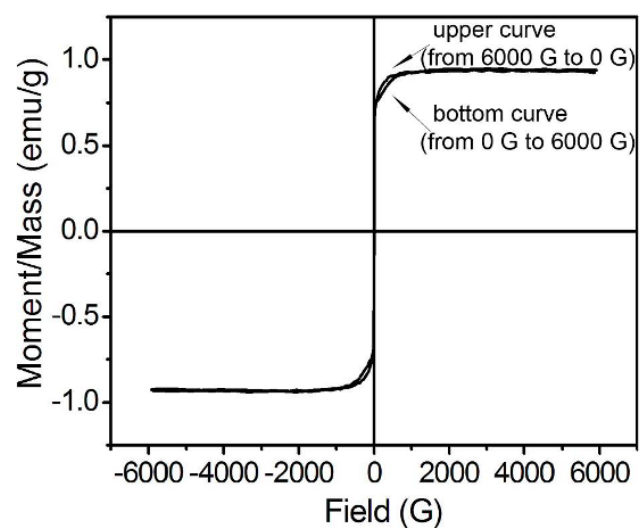

Figure $8 \mid$ Magnetization curve of $\mathrm{Fe}_{3} \mathrm{O}_{4}$ nanosheets suspension (concentration $15 \mathrm{mg} / \mathrm{mL}$ ).

field. The formed one-dimension photonic crystals by the $\mathrm{Fe}_{3} \mathrm{O}_{4}$ nanosheets in aqueous solution under external magnetic field could strongly diffract visible light with different wavelength and display various colors. Diffraction peaks blue shift as the magnetic field increases which could cover the entire visible spectrum. The surface modification increases the dispersibility of the nanosheets and achieves a transformation of ferromagnetism of the particles to a hysteresis loop with much small remanence and coercive force of the particles suspension, which enables the optical response of the colloid solution reproducible and reversible. Excellent magnetic properties of the nanosheets have been obtained with a high saturation magnetization $(82.1 \mathrm{emu} / \mathrm{g})$, low remanence $(13.85 \mathrm{emu} / \mathrm{g})$ and low coercive force $(75.95 \mathrm{Oe})$. The amount of the solvent DEG plays a key role in the formation of the sheet-shaped $\mathrm{Fe}_{3} \mathrm{O}_{4}$ nanostructures. The size of the $\mathrm{Fe}_{3} \mathrm{O}_{4}$ nanoparticles decreases as the ratio of DEG/EG increases in virtue of the strong affinity of DEG to $\mathrm{Fe}^{3+}$ ions and the higher viscosity of the solvent. A transformation of the shape from nanosphere to nanosheet appears when the ratio of DEG/EG is $40 / 0$.

\section{Methods}

Synthesis of polyacrylic acid. $125 \mathrm{~mL}$ Deionized $\mathrm{H}_{2} \mathrm{O}$ and $1.00 \mathrm{~g}$ ammonium persulfate (APS) were added into a three-neck bottle with a dropping funnel and reflux condensing tube. When the APS was dissolved under magnetical stirring, $5.00 \mathrm{~g}$ acrylic acid and $8.00 \mathrm{~g}$ isopropanol were further added. Then the mixture was heated to $65-70^{\circ} \mathrm{C}$, following with the add of a solution containing $40.00 \mathrm{~g}$ acrylic acid, $2.50 \mathrm{~g}$ ammonium persulfate and $40 \mathrm{~mL} \mathrm{DI} \mathrm{H}_{2} \mathrm{O}$ drop by drop through the dropping funnel. Due to the heat released in the polymerization reaction, the temperature of the system would rise and lead to reflux. After all the acrylic acid and APS solution reacted for $30 \mathrm{~min}$, the mixture solution was kept refluxing at $94^{\circ} \mathrm{C}$ for $1 \mathrm{~h}$ and then cooled down.

Synthesis and surface modification of $\mathrm{Fe}_{3} \mathrm{O}_{4}$ particles with sheet-like morphology. Ferrous chloride $\left(\mathrm{FeCl}_{2}\right)$, ferric chloride $\left(\mathrm{FeCl}_{3}\right)$, Sodium Acetate $(\mathrm{NaAc})$ and Polyethylene Glycol (PEG) were dissolved in $40 \mathrm{~mL}$ Diethylene glycol (DEG) under stirring and ultrasonic treatment. The molar ratio of $\mathrm{FeCl}_{2}: \mathrm{FeCl}_{3}: \mathrm{NaAc}$ : $\mathrm{PEG}$ was $1: 1.9: 22: 1.25$. The homogeneous yellow mixture was then transferred to a Teflonlined stainless-steel autoclave and sealed to heat at $220^{\circ} \mathrm{C}$. It is worth noting that the inert gas protection is not necessary due to the closed system and the reducing environment created by DEG. After reaction at $220^{\circ} \mathrm{C}$ for $7 \mathrm{~h}$, the autoclave was cooled down to room temperature. The as-prepared $\mathrm{Fe}_{3} \mathrm{O}_{4}$ particles were washed several times with ethanol and $\mathrm{DI}_{2} \mathrm{O}$ before the surface modification step.

$20 \mathrm{~mL} \mathrm{NaOH}$ solution $(1.00 \mathrm{mM})$ was added into the wet $\mathrm{Fe}_{3} \mathrm{O}_{4}$ precipitation, and then the mixture was ultrasonic treated to disperse the particles into the solution and form the magnetic nanoparticles (MNPs) suspension. $20 \mathrm{~mL}$ PAA solution $(10.0 \mathrm{~g} / \mathrm{L})$ and $1 \mathrm{~mL} \mathrm{FeCl} 3(0.1 \mathrm{~mol} / \mathrm{L})$ were mixed to form an orange PAA-Fe ${ }^{3+}$ complex solution. The MNPs suspension was slowly added into the PAA- $\mathrm{Fe}^{3+}$ complex solution under mechanical stirring. The mixture was ultrasonic treated at $80^{\circ} \mathrm{C}$ for $15 \mathrm{~min}$, and then the precipitate was collected by magnetic separation. After adding $20 \mathrm{~mL} \mathrm{NaOH}$ $(0.05 \mathrm{~mol} / \mathrm{L})$ solution into the precipitate and 10 -s ultrasonic treatment of the mixture, the product was collected again by magnetic separation and washed by DI $\mathrm{H}_{2} \mathrm{O}$ three times. Finally, the magnetic colloid solution was obtained by dispersing the product in a certain amount of $\mathrm{DI} \mathrm{H}_{2} \mathrm{O}$. It should be noted that the particles in the suspension are fairly stable in air and could be stored for months.
Characterization. X-ray diffraction (XRD) patterns were recorded on a Power X-ray Diffractometer (D/Max-IIIA) with $\mathrm{Cu} \mathrm{K} \alpha$ radiation. Transmission electron microscopy (TEM) photographs were taken on a high-resolution transmission electron microscope (HRTEM, JEM-2010) at an accelerating voltage of $200 \mathrm{kV}$. The optical properties of the photonic crystals formed by the magnetic $\mathrm{Fe}_{3} \mathrm{O}_{4}$ nanosheets under external magnetic field, which was controlled by a home-made electromagnet, were collected by using a QE65000-ABS Scientific-grade Optic Fiber Spectrometer (Ocean Optic, Inc.). Room temperature magnetization measurements were performed with a LakeShore Model 7404 vibrating sample magnetometer (VSM).

1. Sun, S., Murray, C., Weller, D., Folks, L. \& Moser, A. Monodisperse FePt nanoparticles and ferromagnetic FePt nanocrystal superlattices. Science 287, 1989-1992 (2000).

2. Miller, M., Prinz, G., Cheng, S.-F. \& Bounnak, S. Detection of a micron-sized magnetic sphere using a ring-shaped anisotropic magnetoresistance-based sensor: a model for a magnetoresistance-based biosensor. Appl. Phys. Lett. 81, 2211-2213 (2002).

3. Jain, T. K., Morales, M. A., Sahoo, S. K., Leslie-Pelecky, D. L. \& Labhasetwar, V. Iron oxide nanoparticles for sustained delivery of anticancer agents. Mol. Pharm. 2, 194-205 (2005).

4. Babes, L., Denizot, B. T., Tanguy, G., Le Jeune, J. J. \& Jallet, P. Synthesis of iron oxide nanoparticles used as MRI contrast agents: a parametric study. J. Colloid Interface Sci. 212, 474-482 (1999).

5. Chourpa, I. et al. Molecular composition of iron oxide nanoparticles, precursors for magnetic drug targeting, as characterized by confocal Raman microspectroscopy. Analyst 130, 1395-1403 (2005).

6. Moser, A. et al. Magnetic recording: advancing into the future. J. Phys. D: Appl. Phys. 35, R157 (2002).

7. Ge, J., Kwon, S. \& Yin, Y. Niche applications of magnetically responsive photonic structures. J. Mater. Chem. 20, 5777-5784 (2010).

8. Jia, C.-J. et al. Iron oxide tube-in-tube nanostructures. J. Phys. Chem. C 111, 13022-13027 (2007).

9. Yang, M., Ma, J., Zhang, C., Yang, Z. \& Lu, Y. General Synthetic Route toward Functional Hollow Spheres with Double-Shelled Structures. Angew. Chem. Int. Ed. 44, 6727-6730 (2005).

10. Wu, M. et al. Magnetic field-assisted hydrothermal growth of chain-like nanostructure of magnetite. Chem. Phys. Lett. 401, 374-379 (2005).

11. Zhong, L.-S. et al. Self-assembled 3D flowerlike iron oxide nanostructures and their application in water treatment. Adv. Mater. 18, 2426-2431 (2006).

12. Xian-Ming, L. \& Jang-Kyo, K. Solvothermal synthesis and magnetic properties of magnetite nanoplatelets. Mater. Lett. 63, 428-430 (2009).

13. Chin, A. B. \& Yaacob, I. I. Synthesis and characterization of magnetic iron oxide nanoparticles via w/o microemulsion and Massart's procedure. J. Mater. Process. Technol. 191, 235-237 (2007).

14. Albornoz, C. \& Jacobo, S. E. Preparation of a biocompatible magnetic film from an aqueous ferrofluid. J. Magn. Magn. Mater. 305, 12-15 (2006).

15. Hee Kim, E., Sook Lee, H., Kook Kwak, B. \& Kim, B.-K. Synthesis of ferrofluid with magnetic nanoparticles by sonochemical method for MRI contrast agent. J. Magn. Magn. Mater. 289, 328-330 (2005).

16. Wan, J., Chen, X., Wang, Z., Yang, X. \& Qian, Y. A soft-template-assisted hydrothermal approach to single-crystal $\mathrm{Fe}_{3} \mathrm{O}_{4}$ nanorods. J. Cryst. Growth 276, 571-576 (2005).

17. Kimata, M., Nakagawa, D. \& Hasegawa, M. Preparation of monodisperse magnetic particles by hydrolysis of iron alkoxide. Powder Technol. 132, 112-118 (2003).

18. Chen, L., Zhao, C., Zhou, Y., Peng, H. \& Zheng, Y. Controlled synthesis of $\mathrm{Fe}_{3} \mathrm{O}_{4}$ nanosheets via one-step pyrolysis of EDTA ferric sodium salt. J. Alloys Compd. 504, L46-L50 (2010).

19. Li, Z. et al. Supercritical Fluid Synthesis of Magnetic Hexagonal Nanoplatelets of Magnetite. J. Am. Chem. Soc. 132, 12540-12541 (2010).

20. Chin, K. C. et al. Large-Scale Synthesis of $\mathrm{Fe}_{3} \mathrm{O}_{4}$ Nanosheets at Low Temperature. J. Phys. Chem. C 111, 9136-9141 (2007).

21. Ge, J., Hu, Y., Zhang, T., Huynh, T. \& Yin, Y. Self-assembly and field-responsive optical diffractions of superparamagnetic colloids. Langmuir 24, 3671-3680 (2008).

\section{Acknowledgments}

This work was supported by the Natural Science Foundation of China (Grant No. 61376014 and No. 60906005), the Guangdong Provincial Natural Science Foundation of China (Grant No. S2013010016010 and No. 9451027501002848) and the Fundamental Research Funds for the Central Universities (Grant No. 11lgpy88 and No. 09ykpy64).

\section{Author contributions}

L.Z. wrote the manuscript and analysed the experimental data, W.Z. provided crucial support for the experiments, Y.Z. and J.L. carried out the experiments, analyzed the experimental data, H.S. supervised the project and H.L. helped writing the main manuscript. 


\section{Additional information}

Competing financial interests: The authors declare no competing financial interests.

How to cite this article: Zhuang, L. et al. Preparation and Characterization of $\mathrm{Fe}_{3} \mathrm{O}_{4}$ Particles with Novel Nanosheets Morphology and Magnetochromatic Property by a Modified Solvothermal Method. Sci. Rep. 5, 9320; DOI:10.1038/srep09320 (2015).

This work is licensed under a Creative Commons Attribution 4.0 International License. The images or other third party material in this article are included in the article's Creative Commons license, unless indicated otherwise in the credit line; if the material is not included under the Creative Commons license, users will need to obtain permission from the license holder in order to reproduce the material. To view a copy of this license, visit http://creativecommons.org/licenses/by/4.0/ 